\title{
Vulnerability and marginality in human services
}

\author{
Mark Henrickson and Christa Fouché, 2017 \\ Routledge, Oxford, UK \\ ISBN 9781472476197, pp. 158, hardback, NZD140.00
}

W hat happens to people when they are classified as vulnerable or marginalised? That is the central question posed by this book. In human services and social policy alike, it has become almost automatic to describe some people as vulnerable or marginalised based on their individual characteristics or belonging to a population group. This book draws together Henrickson and Fouché's diverse research interests to pose questions about the concepts of vulnerability and marginality that emerge as common concerns. They achieve this through focusing on the core human experiences of intimacy and relationship.

The focus for this concentrated volume is restoring the human to human services. The book comprises nine chapters, with the first four chapters exploring how vulnerability and marginality affect people's lives using the overarching themes of intimacy and sexuality. This first part also introduces theoretical contributions to help make sense of the phenomena to emerge from both authors' research. The thoughtful review of both authors' research fields illuminates how notions of vulnerability and marginality have come to dehumanise people who are deemed to be vulnerable or marginalised. Unlike other prominent fields where vulnerability features, this does not include children or child protection. Instead, the dual focus incorporates two adult experiences where people have "changed state" from not being considered vulnerable or marginalised to becoming perceived as such. These research focuses are older people (and specifically older people in residential care) and lesbian, gay, bisexual, trans, intersex, and queer (LGBTIQ) communities, including the specific experiences of HIV+ people.

Henrickson and Fouché draw on structural analyses, in particular that of Loïc Wacquant, to understand the shift from vulnerability and marginality being aspects of the social structure to becoming perceived as characteristics of individuals. These analyses uncover the dehumanising effects that these labels impose on people and their lives in a social environment where risks are perceived as a cost to society. Incorporated throughout is a critique of the dominance of business and efficiency models in human services and the current environment where these are perceived as inevitable or inescapable. The authors explore how these models obscure the workings of power and privilege in turning vulnerability and marginality from structural concepts into characteristics of individuals and communities which, in turn, become thought of as risks that require managing. These discourses of risk are likely to be familiar to anyone working in human services in recent decades.

The book includes a concise and approachable discussion of the rise of neoliberalism as a "how did we get here?" discussion in chapter 4 . This provides the foundation for advocating critique of power and privilege that is exercised through structural mechanisms that are rendered invisible by individualising vulnerability, marginality and, consequently, also risk. 
As becomes apparent in their final chapter, Henrickson and Fouché's challenge for human services and social work is to render that structural power visible again through critical and reflexive practice. A vital contribution, this chapter provides the theoretical and political groundwork for resisting individualisation of structural phenomena prevalent in the neoliberal approach to social policy. Structural analysis of these factors increasingly inscribed onto individuals allows them to be re-ascribed to social structures rather than being perceived as a fault or deficiency of the individual.

When concepts or words become tainted with unwanted meanings, there is a temptation to abandon them as irredeemable from those connotations. Henrickson and Fouché do not suggest abandoning the concepts of vulnerability or marginality entirely. Rather, they conclude the book with an exploration of how to reimagine the concepts and the role of critical reflexive social work and human services. They advocate for sustained attention to the workings of power at structural levels that lead to the detriment caused by application of these concepts to people. They argue that the appropriate response "is not merely to 'help' vulnerable and marginalised individuals and communities, but to critique those with power who create and label those individuals and communities" (p. 132). The challenge for social workers and other human services professionals is to identify how being labelled as vulnerable or marginalised may affect any person and what implications this has for their agency, autonomy, and access to the means for exercising self-determination.

One of the key contributions to the field in this book is its focus on individuals and communities not commonly at the centre of discussions about vulnerability or marginality. The core of this discussion has reach far beyond the focus on older people and LGBTIQ communities. It has implications for any individual or group identified as vulnerable or marginalised.
What the focus on older people and LGBTIQ people does is allow us to see more clearly the effects of notions of vulnerability and marginality when applied to persons. This is especially significant for perceiving the effects on individuals and groups who are routinely or automatically considered vulnerable or marginalised, including children and people with cognitive impairments.

By focusing on groups of adults who have shifted state from being perceived as not vulnerable or not marginalised into a state of being perceived as such, the discussions in this book demonstrate the negative impact of these concepts on people's lives. The examples from their respective research serve to illustrate how people's personhood, autonomy, and selfdetermination become curtailed when they are labelled as vulnerable or marginalised. Labelled with these concepts. people's lives are rendered subject to scrutiny and restriction beyond the scope of the person's limitations. As Henrickson and Fouché argue, this effect is not simply on the individual as a reductionist and isolated unit but infringes on the person's ability and rights to intimacy and relationships subject to the conservative moral qualms of the dominant culture. The most poignant and difficult discussions to read in this regard are the denials of intimacy to older people in residential aged care because of their perceived vulnerability. As many of the cases discussed illuminate, these are the result of legal or organisational policy manifestations of the prudishness of others. Here, where caring, intimate relationships of decades are rendered void by a designation of vulnerability on one of the partners, the dehumanising effects of being labelled as vulnerable are made plain.

The dense theoretical discussion in some sections may offer some challenge for readers who are less practised at reading these types of text. However, the skill of the authors in supporting readers to develop their critical reading and reflection shines 
through in the key questions presented at the end of each chapter. In this respect, the book presents a useful addition to continuing professional development activities as well as a resource for educators and students.

The questions and challenges raised in this book are vital, even central, to social work. It is essential for social workers confronted with organisational focus on risk and risk management in their work to be alert to the effects of deeming an individual or community as vulnerable or marginalised. This must include thinking about people we routinely or automatically think of as vulnerable, including children, and what effect this has on our perceptions of their competence, their personhood and selfdetermination.

What this book achieves is to provoke readers to think carefully about what we harm we might be doing when we refer to a person or group of people as vulnerable or marginalised. Henrickson and Fouché's discussion presents a clear argument that this is not a simple or benign act. If we are serious about social work's responsibility to social justice, empowerment, and tino rangatiratanga, then this is a vital discussion to have. 